\title{
Harzmischung zum Vergolden von Papier, Leder etc. etc. ron
}

Dr. E. Reichardt in Jena.

Die Buchbinder, Galanteriearbeiter u.s.w. gebrauchen gewöhnlich zu dem Vergolden $n i c h t$ metallischer Gegenstände das Eiweiss, welches getrocknet und gepulvert aufbewahrt und bei dem Gebrauche durch wenig Wasser in Lösung oder auch nur in sehr feine Zertheilung gebracht wird.

Im Allgemeinen ist eine Substanz nöthig, welche die Adhäsion des Goldes an den zu vergoldenden Gegenständen vermittelt und könnten hierzu schleimige, leicht trocknende Körper am besten angewendet werden, demnach Leim, Gummi, Pflanzenschleim und das vorzüglich in Gebrauch gezogene Eiweiss in in Wasser löslicher Form. Letzteres hat besonders den Vortheil, dass das darin enthaltene, ganz schwach vorwaltende Alkali die leichtere Verbindung, Adhäsion, an die zu vergoldenden Körper vermittelt und nach dem Austrocknen den Schwankungen der atmosphärischen Feuchtigkeit sich leicht accomedirt, ohne dadurch die Vergoldung zu lockern, wodurch die Beständigkeit derselben bedeutend vermehrt wird.

Geschieht die Vergoldung durch Stempel, um besondere Figuren nach. Wohlgefallen zu erzielen, so ist es wichtig, dass die Lösung von Eiweiss genügend concentrirt sei, um sofort bei dem Drucke des erwärmten Stempels einzutrocknen und an den gedrückten Stellen das Gold so fest, als möglich zu halten. In dem Uebersehen dieser nothwendigen Bedingungen, $d . h$. entweder in der ungenügenden Concentration der Eiweisslösung oder dem zu starken oder geringen Erwärmen des Stempels hat das Misslingen der Vergoldung seinen Grund. 
Vor kurzer Zeit erhielt ich ein Harzpulver zur Untersuchung, welches zu gleichem Zwecke von den Buchbindern als Ersatz des Eiweisses angewendet wird und sehr empfehlenswerth sein soll.

Dass Harze ebenso gut, wie die in Wasser gelösten oben genannten Stoffe die Adhäsion des Goldes bei Anwendung heisser Stempel vermitteln können, versteht sich von selbst, ja sie haben sicher den Vortheil, dass sie sich ganz indifferent gegen die Feuchtigkeit verhalten.

R. Ruding*) wendet zum Golddruck auf seidene und andere Gewebe fein gepulverten und gereinigten Schellack an.

Wie bei der Anwendung von Eiweiss etc. das Gelingen in der Concentration der Lösung und dem Erwärmen des Stempels lag, so hier in dem Grade der Schmelzbarkeit des Harzes oder der Harzmischung. Das Harz muss so leicht schmelzbar sein, dass es sofort bei dem Aufdrücken des Stempels in den flüssigen Zustand übergeht, aber nur in den von dem Stempel berührten Stellen. Harze, welche schon bei der einfachen Annäherung des warmen Stempels schmelzen, würden zu leicht schmelzbar und unbrauchbar sein; zu schwer schmelzbare desgleichen.

Die mir übergebene Mischung von Harzen bestand, nach den Löslichkeitsverhältnissen der Harze in Alkohol, Aether, ätherischen Oelen u. s. w: und den Merkmalen bei steigendem Erhitzen und endlicher Verbrennung etc. untersucht, aus vorwaltendem Copal und etwas Mastix.

4 bis 5 Theile Copal mit 1 Theil Mastix gaben eine bei dem Gebrauche die erforderlichen Eigenschaften besitzende Mischung; übrigens gelingt die Vergoldung auch schon bei Anwendung von reinem Copal.

*) Wagner's Jabresbericht über die Fortschritte der chemischen Technologie. 1856. S. 339. - Repert. of Patent Invent. Sept. 1856. pag. 225. - Polyt. Centralbl. 1856. pag. 1341. 
46 Reichardt, Harzmischung zum Vergolden von Papier etc.

Die Harze müssen auf das Feinste gepulvert und gemischt werden und werden dann mittelst eines Haarpinsels auf den zu vergoldenden Gegenstand aufgestïubt, sogar ein wenig gerieben. Die Vergoldung geht sehr rasch und vorzüglich von statten, sowohl auf Papier, wie Leder.

Da die Mannigfaltigkeit der Harze auch eine grosse Verschiedenheit hinsichtlich des Schmelzgrades bietet, so wird diese Art Vergoldung sehr leicht verallgemeinert und bei sehr verschiedenen Temperaturen gebraucht werden können. 